\title{
Equipo multiprofesional y trabajo interdisciplinario en cuidados paliativos: reflexiones sobre factores contextuales $y$ profesionales que afectan la práctica de Terapia Ocupacional
}

The multi professional team and interdisciplinary work in palliative care: Reflections about contextual and professional factors that affect

Occupational Therapy practice

Equipe multiprofissional e trabalho interdisciplinar nos cuidados paliativos: reflexões sobre fatores contextuais e profissionais que afetam a prática da Terapia Ocupacional

Viviana Marcela León Perilla ${ }^{1}$

Regina Helena Vitale Torkomian Joaquim ${ }^{2}$

Recibido: 13 de noviembre 2019 • Enviado para modificación: 20 de junio 2020 • Aceptado: 6 de agosto 2020 León-Perilla, V.M.L. y Joaquim, R.V.T. (2020). Equipo multiprofesional y trabajo interdisciplinario en cuidados paliativos: reflexiones sobre factores contextuales y profesionales que afectan la práctica de Terapia Ocupacional. Revista Ocupación Humana, 20 (1), 64-81. https://doi.org/10.25214/25907816.949

\section{RESUMEN}

Aunque la atención interdisciplinaria ha sido el modelo filosófico y teórico de trabajo del equipo multiprofesional en los cuidados paliativos, en la práctica, este modelo no asegura una atención conjunta. Este artículo de reflexión presenta diferentes cuestionamientos en torno a la visibilidad y el quehacer de Terapia Ocupacional dentro del equipo de cuidados paliativos. Se rescatan las dinámicas de trabajo de terapeutas ocupacionales brasileñas que han actuado en este campo junto con profesionales de otras áreas, analizando las características del entorno laboral y los factores profesionales que pueden estar influenciando la prestación de una atención profesional conjunta durante el proceso de acompañamiento

\footnotetext{
${ }^{1}$ Terapeuta ocupacional. Magíster en Terapia Ocupacional. Candidata a doctora en Terapia Ocupacional. Hospital Universitario Nacional de Colombia. Bogotá, Colombia. vivianaleonperilla.to@gmail.com iD https://orcid.org/0000-0003-2323-921X

${ }^{2}$ Terapeuta ocupacional. Magíster y doctora en Educación Especial. Docente, Universidad Federal de São Carlos. São Carlos, Brasil. regin@ufscar.br ID https://orcid.org/0000-0003-3700-397X
} 
paliativo. Se espera que este diálogo genere incomodidad y que despierte la motivación de las y los lectores para proyectar y concretar oportunidades que aborden e incorporen la dimensión ocupacional en este tipo de cuidado, cuyo enfoque sea la conservación de la identidad profesional, su visibilidad epistémica y la flexibilización de las competencias intraprofesionales, para una genuina práctica interdisciplinaria.

\title{
PALABRAS CLAVE
}

Terapia Ocupacional, cuidados paliativos, grupo de atención al paciente, ambiente de trabajo colaborativo

\begin{abstract}
Although multidisciplinary care has been the philosophical and theoretical work model of the multi-professional team in palliative care, this model does not ensure a collective attention overall in real life. This reflective article presents multiple questions about the occupational therapist's visibility and duty within the palliative care team. It highlights the work dynamics of Brazilian occupational therapists that have worked together with professionals from other areas. It analyzes the characteristics of the job environment and the professional factors that could be influencing the offer of a joint professional task during the palliative care process. It is expected that this dialogue generates discomfort and that it sparks the readers' motivation to project and specify opportunities that will address and incorporate the occupational dimension in this type of care, whose primary focus is to preserve the professional identity, its epistemic view and the flexibility of the intra-professional competencies to achieve a genuine interdisciplinary practice.
\end{abstract}

\section{KEY WORDS}

Occupational Therapy, palliative care, patient care group, collaborative working environment

\section{RESUMO}

Ainda que a interdisciplinaridade tenha sido o modelo filosófico e teórico de trabalho da equipe multiprofissional nos Cuidados Paliativos, na prática, este modelo não garante uma assistência conjunta na sua totalidade. Este artigo de reflexão apresenta diferentes questionamentos em relação à visibilidade do fazer da Terapia Ocupacional, dentro de uma equipe de Cuidados Paliativos. Resgataram-se as dinâmicas de trabalho de terapeutas ocupacionais brasileiras, que atuam junto com outros profissionais nesta área. Analisaram-se características do ambiente de trabalho, bem como fatores profissionais que influenciam a oferta de um exercício profissional conjunto, durante o processo de acompanhamento paliativo. Espera-se que esse diálogo provoque desconforto e desperte a motivação dos leitores e leitoras, para projetar e concretizar oportunidades que incorporem a dimensão ocupacional neste tipo de cuidado. Oportunidades focadas na conservação da identidade profissional, em sua visibilidade epistêmica, e na conexão flexibilização das competências intraprofissionais, para garantir uma verdadeira prática interdisciplinar.

\section{PALAVRAS-CHAVE}

Terapia Ocupacional, cuidados paliativos, grupo de assistência ao paciente, ambiente de trabalho colaborativo 


\section{Introducción}

Usted importa por lo que es, importa hasta el último momento de su vida y haremos todo lo que esté a nuestro alcance, no solo para que muera de manera pacífica, sino también para que, mientras viva, lo haga con dignidad.

\section{Cicely Saunders}

Esta célebre frase, citada por Robert Twycross en un tributo que rindió a su autora en 2006, enmarca el alma de la filosofía de los cuidados paliativos y rescata los conocimientos y valores, inherentes a las diferentes especialidades de la salud, para ampliar las posibilidades de intervención clínica y terapéutica de las personas con una enfermedad que amenaza la vida.

Cicely Saunders (1918-2005), pionera de los cuidados paliativos y del Movimiento Hospice, definió en los años noventa esa atención integral a partir de la experiencia del dolor, que conceptualizó como dolor total, incluyendo tanto el control de los síntomas físicos como el abordaje y el cuidado de los elementos sociales, emocionales, psíquicos y espirituales (Clark, 2007; Lutz, 2011). Además, estableció el primer espacio de cuidado paliativo, u hospice, equipado con la capacidad científica de un hospital y con un ambiente de hospitalidad, tan cálido como el de un hogar, integrado por un equipo humano técnicamente calificado y afectivamente acogedor (Barbero, 2014) centrado en ofrecer a las personas con enfermedades avanzadas e incurables un lugar donde el dolor y el sufrimiento fuesen aliviados por una cotidianidad ocupacionalmente rica y con énfasis resiliente, a fin de resignificar, a partir de nuevas experiencias, la palabra vida (Pessini y Bertachini, 2006).

Dentro de sus experiencias, Cicely Saunders referenció cómo cuidar el jardín, escribir, pintar, conversar y conformar grupos de arte, de música o de teatro fueron disminuyendo en las personas la ansiedad, la depresión y el miedo generados por los procesos de deterioro, así como también la preocupación de familiares y amigos ante la incertidumbre, al ser incluidos en los procesos terapéuticos, lo que favorecía la búsqueda de sentido y la confianza durante esta etapa final de la vida (Saunders,1972). Esta peculiar belleza del cuidado continúa involucrando a nuevas generaciones de profesionales de la salud y es, quizás, más valorada hoy en día, dada la creciente tensión que producen las múltiples opciones técnicas y tecnológicas que suelen alejar a las personas de una muerte digna, liberadora y coherente con sus dimensiones espiritual, sociocultural y psicológica (Miccinesi et al., 2019).

Actualmente, los cuidados paliativos son definidos por la Asociación Internacional de Hospice y Cuidados Paliativos -IAHPC (por su sigla en inglés), tras un consenso mundial, como "la asistencia activa, holística, de personas de todas las edades con sufrimiento grave relacionado con la salud, debido a una enfermedad severa y especialmente de quienes están cerca del final de la vida" (2018). Esta visión enfatiza en el reconocimiento y el respeto de los valores culturales de las personas y sus familiares, así como en su aplicabilidad en todos los entornos de 
atención clínica por medio de equipos multiprofesionales con formación básica o especializada en esta área para prestar atención en cualquier momento de la enfermedad. Este último punto es relevante, considerando que la evolución de la enfermedad, junto con la implementación de nuevas tecnologías y recursos de soporte para prolongar la vida de las personas (Maciel, 2008) han venido transformado el perfil epidemiológico a nivel de mortalidad y calidad de vida.

Entre tanto, el carácter holístico y multiprofesional de los cuidados paliativos se refiere a entender la complejidad y la multidimensionalidad de los problemas que acarrean las enfermedades crónico-degenerativas, progresivas y potencialmente amenazadoras de la vida (como el cáncer, la insuficiencia cardiaca avanzada, las demencias o las enfermedades neurológicas, entre muchas otras) y las secuelas de los diferentes tratamientos farmacológicos que para ellas se emplean (Organización Mundial de la Salud -OMS, 2018); pues, como refiere Maciel (2008), han generado una nueva interpretación de la muerte, pasando de entenderse como el resultado de un evento puntual a ser considerada un proceso complejo.

Por lo anterior, en esta reflexión consideramos relevante ir más allá de la composición multiprofesional de un equipo de salud en el que intervienen diferentes disciplinas, independientemente del impacto de su interrelación, para resaltar el valor del trabajo interdisciplinario, que como refieren Garzaniti y Romero (2018), necesita de una verdadera comunicación y de la interacción dinámica entre sus miembros para lograr ser inclusivo, igualitario y movilizador de condiciones y aptitudes para asegurar una intervención colectiva y eficaz.

Pensar en ese trabajo interdisciplinario ideal nos obliga a reevaluar el lugar de la Terapia Ocupacional y a pensar, como terapeutas ocupacionales, en el papel que representamos o representaríamos como parte de ese equipo para contribuir en la promoción de la autonomía, la calidad de vida y el confort de una persona y de su familia, quienes, vulnerables y alejados física y afectivamente de aquello que les conecta con su cotidianidad, necesitan resignificar en el hospital el proceso de morir y ser para sentirse partícipes del cuidado, sin prescripciones absolutas, recetas mágicas ni garantías de cura, pero con un acompañamiento que respete las singularidades de la experiencia de finalizar la vida dignamente y con el menor dolor posible.

\section{Sobre el panorama brasileño: dinámica de trabajo de terapeutas ocupacionales en los equipos de cuidados paliativos}

En esta reflexión, por tanto, no nos interesamos en rescatar las experiencias científicas que se centran en el abordaje práctico de la Terapia Ocupacional en los cuidados paliativos, considerando que existen diversas revisiones (Pyle, 2018; Nascimento, 2019) y producciones que explicitan experiencias de intervención (Mckenna y Mellson, 2013; Marston et al., 2015; Baltazar et al., 2016; Eva y Morgan, 2018; Lucchi et al., 2020) y modelos de actuación (American Occupational Therapy Association -AOTA, 2011; Pickens et al., 2016; Essential y McColl, 2019. 
En su lugar, pretendemos enfocarnos en evidencias que ayuden a entender la construcción en torno al trabajo interdisciplinario y los significados de las dinámicas colaborativas para las y los terapeutas ocupacionales en los equipos de cuidados paliativos, desde una realidad cultural más cercana.

Brasil Ilamó nuestra atención porque es el único país de Latinoamérica que, desde una organización gremial, el Consejo Federal de Fisioterapia y Terapia Ocupacional -COFFITO, incorporó una resolución ${ }^{3}$ que rige y define la especialidad de Terapia Ocupacional en contextos hospitalarios, incluyendo la atención en cuidados paliativos, comprendida como: "el ofrecimiento de cuidados terapéuticos ocupacionales, junto con el equipo multiprofesional, a usuarios con condiciones crónico-degenerativas potencialmente fatales (oncológicas y no oncológicas) y que están en tratamiento sin condiciones de modificación de la enfermedad" (COFFITO, 2013, art.4).

La gestión de dicha resolución se realizó al identificar la ausencia de leyes constitucionales relacionadas específicamente con los cuidados paliativos y que la legislación sobre atención oncológica, que contempla acciones en cuidados paliativos, no especifica a la Terapia Ocupacional dentro de las categorías de apoyo multiprofesional, como lo son, por ejemplo, Fisioterapia, Nutrición o Fonoaudiología. Eso supone que la Terapia Ocupacional queda sobreentendida en la definición de rehabilitación exigible conforme a las especialidades (Cordeiro y loshimoto, 2010; De Carlo y Kudo, 2018).
Después de lo mencionado, surgen algunas preguntas: ¿por qué existe una dicotomía entre el paradigma y la práctica en lo que se refiere a la visibilidad y la inclusión del trabajo de Terapia Ocupacional en los equipos de cuidados paliativos?, y desde la perspectiva de las y los terapeutas ocupacionales, ¿cómo se percibe la dimensión del trabajo en equipo?

Para ampliar y ejemplificar las posibles hipótesis frente a estos interrogantes, rescatamos apartados de la producción científica brasileña a partir de la tesis de Maestría en Terapia Ocupacional de una de las autoras (Perilla, 2019), para analizarla temáticamente abordando los factores profesionales y contextuales propuestos por Trembaly et al. (2011) como esenciales en un equipo multiprofesional que realiza atención interdisciplinaria.

Los factores contextuales dan cuenta de las características del entorno laboral e incluyen atributos organizativos y de gestión, así como la naturaleza de la colaboración dentro del equipo y con otros equipos para favorecer la realización oportuna y eficaz de remisiones. Por otro lado, los factores profesionales se relacionan con la esencia de las disciplinas representadas, la capacitación y la experiencia laboral de cada profesional, y la responsabilidad en el desarrollo del equipo (Tremblay et al., 2011). Estos dos factores nos permitirán inferir cómo un grupo de terapeutas ocupacionales perciben la efectividad de sus acciones en el equipo, así como la satisfacción en su trabajo dentro de este.

${ }^{3}$ Resolución 429 del 8 de julio de 2013. 
Será una aproximación contextualizada y delimitada a partir de las evidencias analizadas por Perilla (2019) sobre la percepción del trabajo en equipo de 18 terapeutas ocupacionales que integran equipos de cuidados paliativos oncológicos en hospitales públicos de tres estados ubicados en las regiones Nordeste, Sudeste y Sur de Brasil.

\section{Factores contextuales: estructura de remisión y espacios de trabajo conjunto}

Como ya se ha referido, la colaboración interprofesional es un principio central en la atención de pacientes con enfermedades graves, potencialmente mortales, crónicas o progresivas. En la última década, la Terapia Ocupacional se ha involurado con este tipo de pacientes dando mayor fundamento a las orientaciones y directrices profesionales que validan el quehacer profesional en cuidados paliativos. Este campo de ejercicio se basa en la participación ocupacional y social, y en la comprensión de las consecuencias del declive generado por una enfermedad que amenaza la vida (Mckenna y Mellson, 2013); por tanto, la intervención incide en la calidad de vida, la autonomía, la sensación de control sobre la pérdida de roles y el enriquecimiento ocupacional y espiritual de la cotidianidad, al evitar la evolución de una muerte social antes de la muerte biológica (Marston et al., 2015).

Así lo refieren Pickens et al.:

Los terapeutas ocupacionales reconocen que el desarrollo personal puede seguir ocurriendo hasta los últimos años de la vida y que la par- ticipación en ocupaciones puede ser transformadora, independientemente de la expectativa de vida, y por ello, brindan un servicio único que posibilita la función, confort, seguridad, autonomía, dignidad y participación. (2016, p. 1)

No obstante, la Guía de planificación e implementación de servicios de cuidados paliativos (OMS, 2016) especifica que las necesidades de recurso humano variarán según el tipo de servicio a prestar. Si bien el equipo debe ser interdisciplinario, la cantidad y el nivel de aportes de diferentes profesiones dependerán de la atención prevista y la disponibilidad de personal; siendo posible, pero no indispensable, el abordaje de terapeutas ocupacionales en los contextos domiciliario, hospitalario o comunitario.

En esta reflexión, será el contexto hospitalario el escenario revelador del funcionamiento y la construcción de los equipos de cuidados paliativos, pues este ha sido el espacio predilecto para la implementación de sus principios, pero también porque ha estado definido sistemáticamente por conductas para la prolongación de la vida. De manera contradictoria, estos hechos han generado que se reconsidere e incluya la intervención de diferentes profesionales de la salud, en vista de que el aumento del tiempo por vivir no ha implicado, necesariamente, la mejoría en la calidad de esa vida (Gomes y Othero, 2016).

Perilla (2019) identificó que las terapeutas ocupacionales participantes en su investigación hacen parte de equipos reconocidos por el $60 \%$ de ellas como interdisciplinarios, integrados por entre siete y nueve profesionales de la salud 
de diferentes áreas (figura 1) y que apoyan, principalmente, las consultorías en cuidados paliativos dentro de hospitales públicos de tercer nivel de complejidad.

Así mismo, solo un $40 \%$ de las participantes refirió que se lleven a cabo remisiones e interconsultas. Esta situación las conduce a realizar una identificación autónoma de pacientes para, posteriormente, participar en las reuniones de su equipo (generalmente semanales), o con otros equipos, a fin de solicitar remisión y discusión de los casos (Perilla, 2019). Ello confirma que las medidas protocolarias de remisión en el contexto hospitalario requieren la derivación por parte de Medicina, siendo una de las principales barreras para el uso de los servicios de apoyo y gestión en la dinámica de interconsultas (Barreiro et al., 2016). Así mismo, esto interfiere en la prestación temprana y oportuna de los servicios de Terapia Ocupacional en los cuidados paliativos, en medio de un sistema de referencia que depende de la articulación, el adecuado flujo de la información y el apoyo logístico interprofesional.

Aunque las entrevistadas indicaron que Medicina y Enfermería están entre las tres profesiones que mayor número de remisiones realizan, estas ejecutan pocas intervenciones conjuntas con Terapia Ocupacional. El trabajo colectivo indicado por el $94 \%$ de las participantes está vinculado con otras profesiones, como Psicología $(55,5 \%)$ y Fisioterapia (55,5\%) (figura 2$)$.

Figura 1. Integrantes de los equipos de cuidados paliativos oncológicos, según las terapeutas ocupacionales entrevistadas por Perilla (2019).

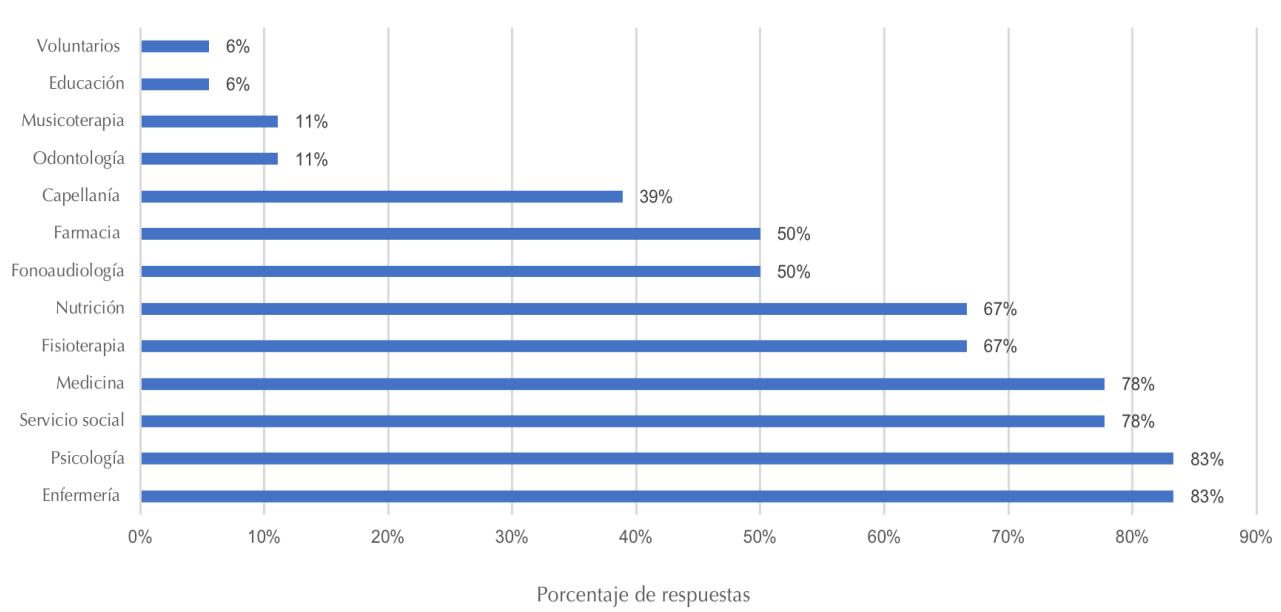

Fuente: adaptado de Perilla (2019). 
Figura 2. Dinámica de remisiones y trabajo colaborativo entre Terapia Ocupacional y demás profesiones del equipo de cuidados paliativos oncológicos, según las terapeutas ocupacionales entrevistadas por Perilla (2019).

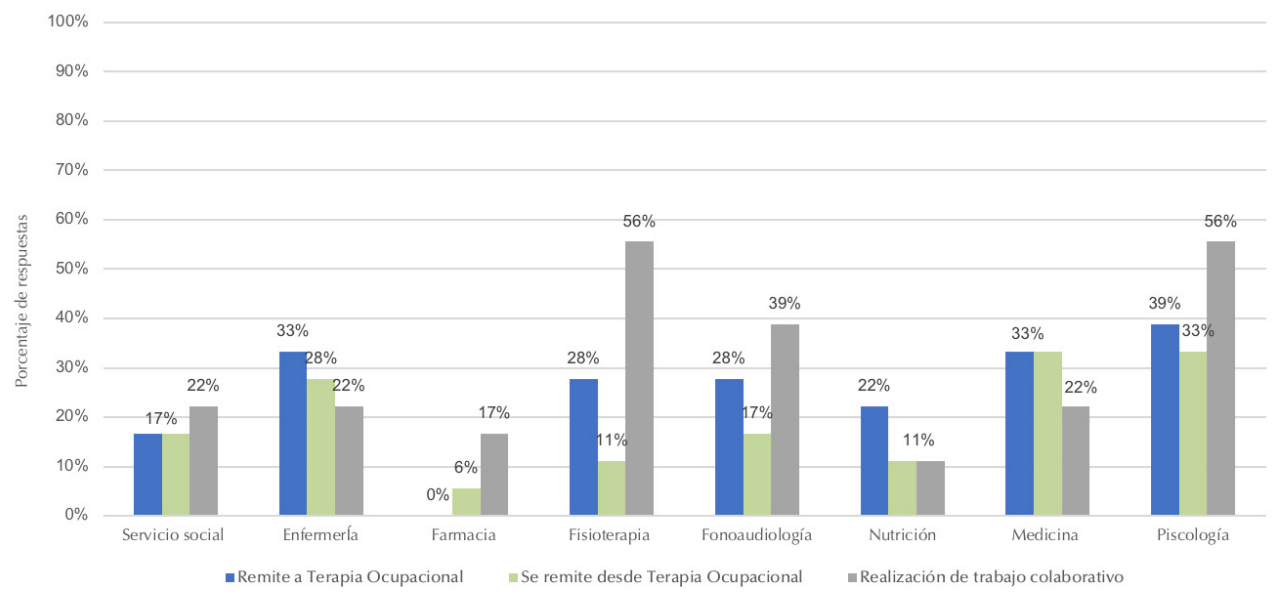

Fuente: adaptado de Perilla (2019).

Lo anterior permite ver que un sistema de derivación multiprofesional no asegura la realización de procesos de atención en salud interdisciplinarios, ni que se comprenda en su totalidad la actuación colectiva de quienes integran el equipo; por el contrario, puede acentuar identidades jerárquicas de cada rol, según su reconocimiento en el contexto sanitario (Barbero, 2014).

En la literatura sobre cuidados paliativos acontece lo mismo. El papel de la Medicina y la Enfermería se presenta detalladamente, lo que es menos frecuente para los roles de corte terapéutico y psicosocial, o se centra en la relación entre el personal de Medicina y Enfermería o en el trabajo en equipo entre especialistas en oncología, cirugía, patología, etc. (Tremblay et al., 2011). Esto evidencia que, en la distribución del trabajo en el equipo interdisciplinario de cuidados paliativos, siguen teniendo un peso di- ferencial los aspectos médicos, psicológicos, sociales, espirituales y ocupacionales. Así mismo, en la identificación formal e informal de los roles hay una baja tendencia al abordaje conjunto de la persona, en una modalidad de cuidado caracterizada por su enfoque humanizado y holístico.

Todo ello indica la necesidad de realizar más investigaciones que permitan esclarecer las identidades y dinámicas en el trabajo interprofesional; comprender los roles para proporcionar servicios efectivos y atención de alta calidad a las y los pacientes, y aportar al desarrollo de competencias clínicas que contribuyan a la satisfacción de las personas en el final de sus vidas (Fukui., et al, 2019). En medio de tantos aspectos por identificar, ¿desde qué perspectiva están siendo contempladas las necesidades de pacientes, familiares y cuidadores en los cuidados paliativos? 
Se sabe que en los marcos institucionales sanitarios, buena parte de la toma de decisiones se ajusta a la necesidad imperiosa de seguir un plan de acción acorde con el diagnóstico y el tratamiento médico, quirúrgico o farmacológico (Lanceley et al., 2008), olvidando identificar y reconocer a la persona, rescatar su historia de vida, escuchar y atender a las necesidades relacionadas, por ejemplo, con sus limitaciones funcionales, la pérdida de autonomía y participación social, o la ruptura y el empobrecimiento de su vida cotidiana. Sin duda, tales decisiones influyen en la participación de Terapia Ocupacional en el acompañamiento de todo el proceso de evolución de una enfermedad crónica, degenerativa y sin perspectiva de cura.

A lo anterior se suma que otras profesiones tienen reservas frente al uso de ocupaciones y actividades terapéuticas en la atención paliativa (Ivy, 2016), basadas en la preocupación porque, al aumentar el nivel de actividad y participación de la persona, puedan incrementarse el dolor o la fatiga. Pero, ¿serán las actividades y el uso de ocupaciones las únicas estrategias y herramientas de trabajo de las y los terapeutas ocupacionales? ¿Se trata solamente de preocupación o hay en el fondo falta de comunicación y de esclarecimiento de las competencias profesionales?

Favorecer una cultura organizativa y de gestión que promueva una mayor claridad en los roles y una suficiente compenetración en los espacios de interacción, podría ayudar a derribar obstáculos inherentes al hecho de lidiar con conocimientos y perspectivas distintas, así como a superar la limitante clasificación de las intervenciones en farmacológicas y no farmacológicas; pues más allá de estas modalidades, se necesita coordinar la atención para que las personas y sus familias puedan transitar sin interrupciones por una atención integral e integrada (Witt Sherman et al., 2017).

A modo de ejemplo, los casos estudiados por Fukui et al. (2019) muestran que los programas multidisciplinarios de intervención al final de la vida mejoran la confianza de los equipos en su capacidad de colaborar para apoyar a las personas que atraviesan por tales circunstancias, cuando tienen en cuenta criterios de comunicación asertiva con profesionales de diferentes especialidades y conocen sus rostros y características personales. Es, pues, una invitación a conocer el proceso profesional que desarrolla cada integrante del equipo, con su particularidades culturales, étnicas, generacionales, religiosas, de género, para llegar a una identificación constructiva de las brechas en la articulación que permita enfocarse en la compenetración de las competencias y asegurar un abordaje integral, compasivo y rentable, fundamental en los cuidados paliativos.

\section{Factores profesionales: habilidades y capacidades para el trabajo en equipo de cuidados paliativos}

Existe evidencia de que las y los profesionales de la salud tienen aún una comprensión limitada sobre la función y el propósito de la Terapia Ocupacional en los cuidados paliativos; esto afecta el número de remisiones oportunas (Hammill et al., 2014; Phipps y Cooper, 2014; Knecht-Sabres et al., 2019; Chow y Pickens, 2020) y las posibilidades de antici- 
par e identificar barreras y limitaciones funcionales, así como sus implicaciones a nivel ocupacional, por lo cual estas raramente se documentan y se registran (Cheville et al., 2008). En consecuencia, las discusiones del equipo giran alrededor de información meramente física para actualizar el cuadro clínico; en este punto, las contribuciones desde otras perspectivas se tornan extrañas y no se incluyen entre los objetivos del trabajo mancomunado. Así, las necesidades ocupacionales de las personas se ven marginadas debido a la falta de potencial de rehabilitación, en lugar de reorientarse hacia la participación con apoyo frente a las demandas reales del final de la vida. Esto sugiere la necesidad de cambiar el paradigma de salud y bienestar que influencia las relaciones interdisciplinarias (Chow y Pickens, 2020) y determinar la posibilidad de anticipar una atención temprana y oportuna por parte de Terapia Ocupacional. Sobre lo anterior, Eva y Morgan refieren:

La capacidad de los terapeutas ocupacionales para usar toda la gama de habilidades y conocimientos para ayudar a los pacientes a vivir bien durante su proceso de declive parece estar significativamente influenciado por las expectativas de sus colegas y por la comprensión de su papel. (2018, p.967)

Así las cosas, ¿qué motiva las remisiones a Terapia Ocupacional dentro del equipo de cuidados paliativos?, ¿se identifican competencias compartidas entre profesiones o se trata de un trabajo en el que estas solo se suman? Para estructurar un raciocinio, partimos de la percepción de las terapeutas ocupacionales entrevistadas por Perilla (2019) sobre por qué y con que frecuencia otras y otros profesionales realizan remisiones a sus servicios.

A pesar de la percepción sobre el bajo número de remisiones (menos del $40 \%$ de integrantes de los equipos remite a Terapia Ocupacional), es alentador ver que entre los principales motivos para hacerlo estén el rescate de la participación ocupacional en el ambiente hospitalario, así como el apoyo en las actividades productivas y de tiempo libre, lo que se encuadra dentro del uso terapéutico de ocupaciones y actividades (tabla 1). También es interesante, porque esto contradice los resultados de algunas investigaciones internacionales que señalan la dificultad de las y los profesionales en cuidados paliativos para centrarse en la participación en ocupaciones significativas (Keesing y Rosenwax, 2011; Knecht-Sabres et al., 2019), siendo más frecuentes las remisiones para prescripción de tecnología de asistencia, realización de adaptaciones en el ambiente físico, terapia de mano, ejercicio físico y prueba muscular/articular de mano, confección y prescripción de férulas (Phipps y Cooper, 2014; Knecht-Sabres et al., 2019).

Ese énfasis en el desempeño ocupacional, no en la participación ocupacional para la comprensión del proceso de la muerte y en la influencia del medio ambiente social y cultural sobre el compromiso ocupacional al final de la vida (Chow y Pickens, 2020), dialoga con la incertidumbre respecto a la posibilidad de desplegar por completo el rol de la Terapia Ocupacional en acciones colectivas que implican educación, ayuda en la comunicación de noticias difíciles, mediación de conflictos y asesoría a otros profesionales (tabla1). En este contexto, se valora la divulgación de las 
competencias profesionales a partir de una visión tradicional de la rehabilitación, en lugar de centrarse en la capacidad de ayudar a las personas a vivir con calidad de vida hasta su muerte.

Es necesario, por consiguiente, flexibilizar, delegar y compartir el liderazgo para aumentar las oportunidades de acción y asesoramiento por parte de todos los miembros del equipo; en la medida que la atención al final de la vida crece en demanda y complejidad, el énfasis en la participación sostenida en roles e intereses significativos resulta fundamental para continuar garantizando el ser, pertenecer y hacer de forma digna, incorporando el uso de la actividad y la participación ocupacional como puentes que pueden ayudar a derrumbar el cerco de silencio entre la persona, su familia y las y los profesionales.

Tabla 1. Motivos de remisión a Terapia Ocupacional por parte del equipo de cuidados paliativos.

\begin{tabular}{|c|c|c|c|c|c|c|c|c|c|c|c|c|}
\hline \multirow[b]{2}{*}{ Grupos temáticos } & \multirow[b]{2}{*}{$\begin{array}{l}\text { Motivos de remisión a Terapia } \\
\text { Ocupacional }\end{array}$} & \multicolumn{9}{|c|}{ Número de respuestas por profesión } & \multirow[b]{2}{*}{$\begin{array}{c}\text { Total } \\
\mathrm{N}\end{array}$} & \multirow[b]{2}{*}{$\begin{array}{c}\text { Total } \\
\%\end{array}$} \\
\hline & & 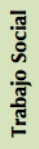 & 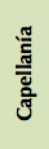 & 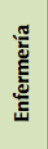 & 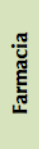 & 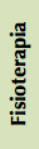 & 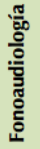 & & 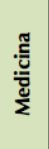 & $\begin{array}{l}\frac{\pi}{00} \\
\frac{0}{\circ} \\
\frac{0}{20} \\
\frac{0}{2}\end{array}$ & & \\
\hline \multirow{2}{*}{ Evaluación } & $\begin{array}{l}\text { Evaluación de aspectos sensorio-motores } \\
\text { y cognitivos }\end{array}$ & 3 & 0 & 3 & 0 & 11 & 7 & 2 & 14 & 5 & 45 & $27 \%$ \\
\hline & $\begin{array}{l}\text { Evaluación del grado de independencia } \\
\text { en el desempeño ocupacional }\end{array}$ & 3 & 0 & 6 & 1 & 12 & 5 & 2 & 12 & 5 & 46 & $28 \%$ \\
\hline \multirow{3}{*}{$\begin{array}{l}\text { Uso terapéutico de } \\
\text { ocupaciones y } \\
\text { actividades }\end{array}$} & $\begin{array}{l}\text { Rescatar participación ocupacional en el } \\
\text { ambiente hospitalario }\end{array}$ & 7 & 3 & 8 & 1 & 6 & 5 & 6 & 9 & 12 & 57 & $35 \%$ \\
\hline & Favorecer actividades de ocio y laborales & 7 & 4 & 10 & 2 & 0 & 7 & 8 & 11 & 11 & 60 & $37 \%$ \\
\hline & Exploración de nuevos pasatiempos & 2 & 3 & 9 & 3 & 4 & 1 & 3 & 5 & 5 & 35 & $21 \%$ \\
\hline \multirow{3}{*}{$\begin{array}{l}\text { Educación y } \\
\text { entrenamiento }\end{array}$} & $\begin{array}{l}\text { Simplificación y entrenamiento de AVD, } \\
\text { ABVD y AIVD }\end{array}$ & 0 & 0 & 3 & 1 & 6 & 6 & 1 & 6 & 3 & 26 & $16 \%$ \\
\hline & $\begin{array}{l}\begin{array}{l}\text { Entrenamiento de estrategias para control } \\
\text { de síntomas }\end{array} \\
\end{array}$ & 1 & 1 & 7 & 0 & 4 & 4 & 2 & 7 & 5 & 31 & $19 \%$ \\
\hline & Apoyo en el ajuste del estilo de vida & 3 & 1 & 6 & 0 & 3 & 3 & 3 & 5 & 7 & 31 & $19 \%$ \\
\hline \multirow{4}{*}{$\begin{array}{l}\text { Uso de métodos } \\
\text { preparatorios }\end{array}$} & Dotación de equipo de apoyo & 1 & 1 & 3 & 0 & 3 & 2 & 1 & 4 & 2 & 17 & $10 \%$ \\
\hline & Confección de órtesis de posicionamiento & 1 & 0 & 2 & 0 & 0 & 2 & 1 & 6 & 2 & 14 & $8 \%$ \\
\hline & Prescripción de tecnología de asistencia & 2 & 0 & 4 & 0 & 9 & 2 & 0 & 4 & 0 & 21 & $12 \%$ \\
\hline & Adaptaciones en el ambiente & 3 & 0 & 6 & 0 & 6 & 5 & 1 & 7 & 7 & 35 & $21 \%$ \\
\hline \multirow{5}{*}{$\begin{array}{c}\text { Apoyo psicosocial al } \\
\text { paciente, familia y } \\
\text { equipo }\end{array}$} & $\begin{array}{l}\text { Organización de proyectos en el final de } \\
\text { la vida }\end{array}$ & 2 & 2 & 3 & 0 & 2 & 3 & 2 & 8 & 5 & 27 & $16 \%$ \\
\hline & $\begin{array}{l}\text { Auxilio en la comunicación de noticias } \\
\text { difíciles }\end{array}$ & 0 & 2 & 4 & 0 & 2 & 1 & 0 & 7 & 5 & 21 & $12 \%$ \\
\hline & Mediación de conflictos & 1 & 2 & 6 & 0 & 0 & 1 & 0 & 4 & 3 & 17 & $10 \%$ \\
\hline & Acompañamiento al familiar/cuidador & 3 & 2 & 9 & 1 & 4 & 2 & 2 & 6 & 6 & 35 & $21 \%$ \\
\hline & Asesoría y apoyo a otros profesionales & 0 & 2 & 3 & 0 & 1 & 1 & 1 & 1 & 2 & 11 & $6 \%$ \\
\hline
\end{tabular}

Fuente: adaptado de Perilla (2019). 
Por otra parte, es claro que no siempre se está en capacidad de responder a las demandas del equipo; con frecuencia, la acción profesional se limita a remitir a la persona a otra especialidad que se considere más adecuada en ese momento (Portela y Galheigo, 2015). Al identificar las competencias profesionales de Terapia Ocupacional, sería mayor la remisión y la delegación de decisiones sobre dilemas relacionados con las actividades que podrían tener sentido al final de la vida y cuál sería la mejor manera de llevarlas a cabo, de acuerdo con los deseos de las personas. Un gran desafío, entonces, es encontrar un liderazgo compartido en el que la dificultad de hablar de igual a igual se transforme en la búsqueda de un lenguaje común. En la misma perspectiva, Oudshoorn destaca que:

Este trabajo no se da por la sumatoria de disciplinas, sino por una dinámica particular en la cual cada miembro aporta sus saber disciplinar y su mirada particular. Si el hacer consensuado es terapéutico, entonces la interdisciplinariedad otorga significado a las intervenciones. Las bases conceptuales de cada disciplina se apoyan y, en cierta medida, se desdibuja el territorio práctico de cada profesional para enfocar los esfuerzos terapéuticos en los resultados esperados. $(2015$, p.91)

Además, por tornarse en un proceso interdependiente, implica también que la o el terapeuta ocupacional se sintonice con el equipo a partir de una actitud asertiva con la cual abogue y haga de sus competencias una construcción diaria responsable, autónoma y cooperativa.

Entre tanto, la percepción frecuente de que se carece de la formación nece- saria para ejercer con suficiencia en cuidados paliativos (Keesing y Rosenwax, 2011; Breen et al., 2013; Hammill et al., 2014) ha minado la autoestima profesional de las y los terapeutas ocupacionales (Portela y Galheigo, 2015), lo cual se refleja en la baja oferta laboral para esta área y en el escaso número de profesionales ejerciendo actualmente en equipos de cuidados paliativos (Essential y McColl, 2019).

En diversos documentos, el uso pleno de las habilidades interpersonales y de la comunicación se han considerado como elementos básicos para realizar un abordaje competente en los procesos terapéuticos de acompañamiento al final de la vida (College of Occupational Therapists, 2011). No obstante, la compenetración entre el saber y el significado que se da a ese conocimiento en la resolución de problemas han permitido a las y los terapeutas ocupacionales responder de manera oportuna y adecuada a las complejas necesidades de las personas en cuidados paliativos

Como se presenta en la figura 3, dado que valoran las historias de vida de las personas, las y los terapeutas ocupacionales pueden manifestar sensibilidad, empatía, escucha activa y receptividad frente a sus emociones, responsabilidades, deseos y preferencias. Ahora bien, es en el trabajo en equipo donde se pueden concretar y gestionar la preservación de la dignidad, la privacidad, la autonomía y la autodeterminación, toda vez que la comunicación esté permeada por la claridad conceptual, la confianza técnica, el pensamiento flexible y la entrega (Perilla, 2019). Retomando la solicitud del paciente David Tasman a Cicely Saunders, de escuchar "lo que está en tu 
mente y en tu corazón" ${ }^{\prime 4}$, se trata de hacer algo por el cuidado de las personas que están finalizando su vida (Strang, 2004) basado en un constructo que combine aquello que nace de la disciplina académica con lo que surge de la motivación personal y de la fe.

Así, aunque los valores personales y las habilidades emocionales configuren y favorezcan la humanización del trabajo y el aprendizaje a través de la experiencia, es necesario estar en sin- tonía con la visión taxonómica y filosófica del cuidado paliativo.

Como se dice coloquialmente, cada desafío es una nueva oportunidad, de manera que frente a la ausencia de modelos que se centren en aspectos paliativos en la formación profesional en Terapia Ocupacional en Latinoamérica, surge la responsabilidad de evaluar y actualizar constantemente nuestro quehacer con miras a proyectar oportunidades y nuevos caminos paradig-

Figura 3. Habilidades percibidas como necesarias para la práctica en cuidados paliativos por parte de las terapeutas ocupacionales entrevistadas por Perilla (2019).

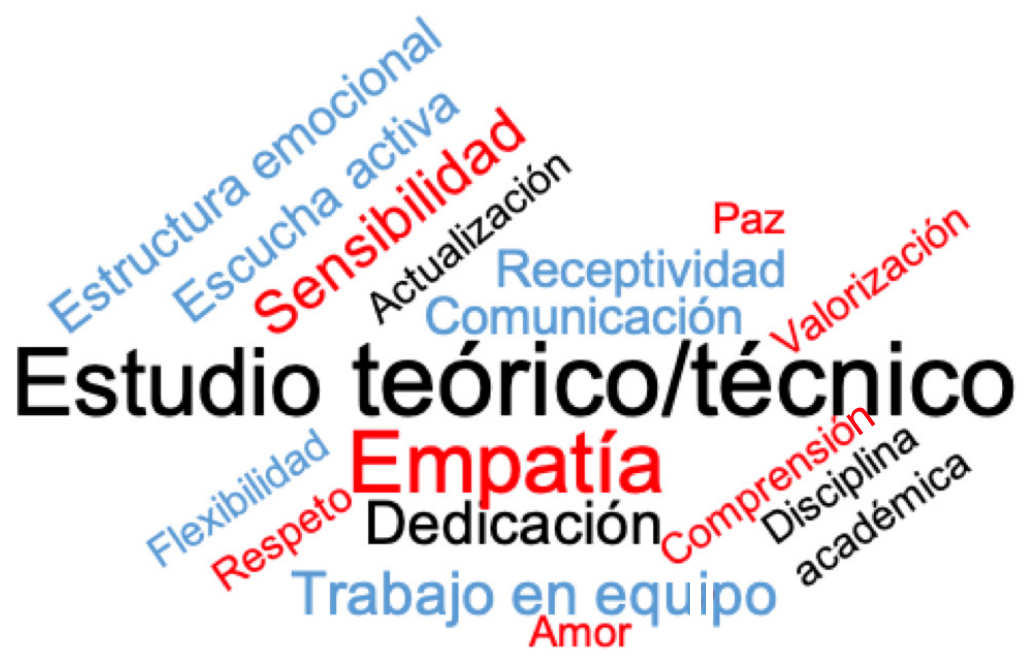

Estrategias de actuación

Fuente: adaptado de Perilla (2019).

${ }^{4}$ En 1947, Cicely Sanders inició su primer trabajo como trabajadora social en el Saint Thomas Hospital, en el área pacientes con cáncer. Allí empezó a desarrollar una intensa aspiración, confianza e indagación espiritual. En ese mismo año conoció a David Tasman, quien estaba internado en el Saint Thomas y le quedaba poco tiempo de vida. Se acercaron cada vez más y sostenían largas charlas sobre temas religiosos y sobre cómo deberían ser cuidadas las personas cuando están muriendo y atravesando por situaciones de sufrimiento y dolor, muchas veces en soledad. Un día, Tasman le pidió a Sanders escuchar algo reconfortante, ella intentó leerle un salmo de la Biblia. Tasman la interrumpió diciéndole: "solo quiero lo que está en tu mente y en tu corazón". Ese pedido se transformó con el tiempo en el espíritu de los cuidados paliativos (Wainer, 2003). 
máticos para abordar e incorporar la dimensión ocupacional en este tipo de cuidado, buscando la conservación de la identidad profesional, la visibilidad epistémica, la flexibilización de las competencias intraprofesionales y el fomento del trabajo interdisiciplinario.

\section{Conclusiones}

Esperamos que este artículo haya despertado la curiosidad y genere nuevos cuestionamientos en torno a la visibilidad y el quehacer de la Terapia Ocupacional en los equipos de cuidados paliativos, como una profesión mediadora y que está en capacidad de identificar, exponer, amparar y atender las necesidades ocupacionales de las personas con una enfermedad que amenaza sus vidas.

El trabajo interdisciplinario ha sido el modelo que mejor describe la dinámica de los equipos en los que participan diferentes profesionales en los cuidados paliativos, no obstante, no asegura en su totalidad una atención conjunta. En el caso de la incorporación de Terapia Ocupacional a esta abordaje, son diversos los factores contextuales y profesionales que pueden influenciar la prestación continua de la atención durante el proceso de acompañamiento, desde la reciente vinculación de esta profesión a la atención paliativa, un sistema de referencia y contrarreferencia basado exclusivamente en el criterio médico, hasta la falta de comprensión del equipo de su quehacer.

Sin embargo, existe una tendencia favorable a ganar espacio profesional en el equipo de cuidados paliativos, sin tener que dejar atrás nuestro objeto de trabajo: la participación ocupacional. Las y los te- rapeutas ocupacionales hemos emprendido este camino manteniendo nuestro trabajo en un perspectiva multifocal e integral, específicamente con profesionales de otras especialidades terapéuticas y psicosociales con quienes, con frecuencia, compartimos la atención conjunta y las discusiones sobre los casos.

Todavía son muchos los desafíos, en el caso de Latinoamérica, específicamente en el contexto brasileño, la Terapia Ocupacional está construyendo un cuerpo de conocimientos basado en el aumento del registro sistemático de las acciones que desarrolla, también, a través de espacios organizativos de incidencia política. Se necesita un cambio en la educación sanitaria y clínica. Se requiere práctica, comenzando por la posibilidad de que estudiantes y profesionales participen de espacios de aprendizaje interactivo con otras profesiones. Es fundamental que ese movimiento permee el ámbito formativo, con el fin de consolidar experiencias y oportunidades laborales exitosas que reflejen la convicción en el potencial de nuestro trabajo en esta área, nuestra capacidad de articularnos en los equipos interdisciplinarios y el respaldo en los conceptos y la filosofía de los cuidados paliativos. 


\section{Referencias}

American Occupational Therapy Association [AOTA] (2011). The role of occupational therapy in end-of-life care. American Journal of Occupational Therapy, 65(Suppl.), S66--S75. https://doi.org/10.5014/ ajot.2011.65S66

Barbero, J. (2014). El trabajo en equipo de los equipos multidisciplinares de cuidados paliativos: análisis de la realidad española [tesis doctoral, Universidad Autónoma de Barcelona]. Depósito Digital de Documentos de la UAB. https://ddd.uab. cat/record/127161

Baltazar, H. M. C., Pestana, S. C. C. y Santana, M. R. R. (2016). Contributo da intervenção da terapia ocupacional nos cuidados paliativos. Cadernos Brasileiros de Terapia Ocupacional, 24(2), 261-273. https://doi. org/10.4322/0104-4931.ctoAO0692

Barreiro, G., Zanella, F. A., Rosa, K. G. D., Calvett, R., Senandes, L. S., Vizzotto, M. D., ... y Lima, V. S. D. (2016). O impacto de ações assistenciais na percepção da qualidade do Sistema Único de Saúde (SUS), Brasil: um estudo transversal. Rev. bras. cir. plást, 31(2), 242-245.

Strang, P. (2004). Book review: Watch with me. Inspiration for a life in hospice care. PaIliative Medicine, 18(7), 672-672. https:// doi.org/10.1177/026921630401800723

Breen, L. J., Fernandez, M., O'Connor, M. y Pember, A.J. (2013). The preparation of graduate health professionals for working with bereaved clients: An Australian perspective. OMEGA - Journal of Death and Dying, 66(4), 313-332. https://doi. org/10.2190/om.66.4.C
Cheville, A. L., Beck, L. A., Petersen, T. L., Marks, R. S. y Gamble, G. L. (2008). The detection and treatment of cancer-related functional problems in an outpatient setting. Supportive Care in Cancer, 17(1), 61-67. https://doi.org/10.1007/s00520008-0461-x

Chow, J. K. y Pickens, N. D. (2020). Measuring the efficacy of occupational therapy in end-of-life care: A scoping review. American Journal of Occupational Therapy, 74(1), https://doi.org/10.5014/ ajot.2020.033340

Clark, D. (2007). From margins to centre: a review of the history of palliative care in cancer. The lancet Oncology, 8(5), 430-438. https://doi.org/10.1016/s14702045(07)70138-9

College of Occupational Therapists (2011). The route to success in end of life care-achieving quality for people with learning disabilities. Achieving quality for occupational therapy. https:// www.rcot.co.uk/sites/default/files/Route-to-success-Occupational-Therapy $\% 20$ \%281\%29.pdf

Conselho Federal de Fisioterapia e Terapia Ocupacional [COFFITO] (2013, 8 de julio). Resolução $n^{\circ} 429$, de 08 de julho de 2013, reconhece e disciplina a especialidade de Terapia Ocupacional em Contextos Hospitalares, define as áreas de atuação e as competências do terapeuta ocupacional especialista em contextos hospitalares e das outras providências. http://www.crefito2.gov.br/legislacao/ resolucoes-coffito/resolucao-429--de-08de-julho-de-2013-1670.html 
Cordeiro, J. J. R. y Joshimoto, M. T. A. (2010) Organização de serviços de terapia ocupacional - gestão a partir de dados e indicadores. En M. Othero (ed.), Terapia Ocupacional práticas em Oncologia (pp.123-140). Roca.

De Carlo, M. M. y Kudo, A. M. (2018). Terapia ocupacional em contextos hospitalares e cuidados paliativos. Payá.

Essential, H.H.y McColl, M. A. (2019). A model for occupation-based palliative care. Occupational Therapy In Health Care, 33(1), 108-123. https://doi.org/10.1080/0 $\underline{7380577.2018 .1544428}$

Eva, G. y Morgan, D. (2018). Mapping the scope of occupational therapy practice in palliative care: A European Association for Palliative Care cross-sectional survey. PaIliative Medicine, 32(5), 960-968. https:// doi.org/10.1177/0269216318758928

Fukui, S., Fujita, J., Ikezaki, S., Nakatani, E. y Tsujimura, M. (2019). Effect of a multidisciplinary end-of-life educational intervention on health and social care professionals: A cluster randomized controlled trial. Plos One, 14(8), 1-12. https://doi. org/10.1371/journal.pone.0219589

Garzaniti, R. y Romero, P. M. (2018). Atención integral de la salud: la interdisciplina como herramienta fundamental. Anuario Temas en Psicología, 4, 275-294. https:// revistas.unlp.edu.ar/AnuarioPsicologia/article/view/8465

Gomes, A. L. Z. y Othero, M. B. (2016). Cuidados paliativos. Estudos Avançados, 30(88), 155-166. https://doi.org/10.1590/ s0103-40142016.30880011

Hammill, K., Bye, R. y Cook, C. (2014). Occupational therapy for people living with a life-limiting illness: A thematic review. British Journal of Occupational Therapy, 77(11), 582-589. https://doi.org/10.4276/ $\underline{030802214 \times 14151078348594}$

International Association for Hospice and Palliative Care [IAHPC]. (2018). Global consensus based palliative care definition. International Association for Hospice and Palliative Care. https:// hospicecare.com/what-we-do/projects/ consensus-based-definition-of-palliative-care/definition/

Ivy, M. (2016). The role of occupational therapy in palliative care: is it perceived to be beneficial by the patient and family? [Tesis doctoral, Texas Woman's University]. Repository TWU. http://hdl.handle. net/11274/9652

Keesing, S. y Rosenwax, L. (2011). Is occupation missing from occupational therapy in palliative care? Australian Occupational Therapy Journal, 58(5), 329-336. https://doi.org/10.1111/j.1440$\underline{1630.2011 .00958 . x}$

Knecht-Sabres, L. J., Weppner, A., Powers, C. y Siesel, B. (2018). Do health-care professionals really understand the role of occupational therapy in hospice care? American Journal of Hospice and Palliative Medicine, 36(5), 379-386. http://doi. org/10.1177/1049909118812858

Lanceley, A., Savage, J., Menon, U., y Jacobs, I. (2008). Influences on multidisciplinary team decision-making. International Journal of Gynecologic Cancer, 18(2). 215-222. http://dx.doi.org/10.1111/j.1525-1438.2007.00991.x

Lucchi, E., Caiata-Olgiati, G., Bonomi, M. y Cembrani, F. (2020). Occupational therapy and palliative care. En C. Pozzi, A. 
Lanzoni, M. Graff y A. Morandi (eds.), Occupational Therapy for Older People (pp. 125-144). Springer.

Lutz, S. (2011). The history of hospice and paIliative care. Current problems in cancer, 35(6), 304-309. https://doi.org/10.1016/j. currproblcancer.2011.10.004

Maciel, M.G. (2008). Definições e princípios. En Conselho Regional de Medicina do Estado de São Paulo -Cremesp, Cuidado paliativo (pp.15-32). Cremesp.

Marston, C., Agar, M. y Brown, T. (2015). Patients' and caregivers' perceptions of occupational therapy and adapting to discharge home from an inpatient palliative care setting. British Journal of Occupational Therapy, 78(11), 688-696. https:// doi.org/10.1177/0308022615586417

McKenna, J. y Mellson, J.A. (2013). Emotional intelligence and the occupational therapist. British Journal of Occupational Therapy, 76(9), 427-430. https://doi.org/ $\underline{10.4276 / 030802213 \times 13782044946382}$

Miccinesi, G., Caraceni, A., Garetto, F., Zaninetta, G., Bertè, R., Broglia, C. y Maltoni, M. (2019). The path of Cicely Saunders: The "peculiar beauty" of palliative care. Journal of Palliative Care, 35(1), 3-7. https:// doi.org/10.1177/0825859719833659

Nascimento, W. M. S. D. (2019). Produção científica em cuidados paliativos e terapia ocupacional: revisão de escopo [Tesis de Especialización, Universidade Federal de Paraiba]. Repositorio UFPB. https://repositorio.ufpb.br/jspui/hand$\underline{\text { le/123456789/17460 }}$

Organización Mundial de la Salud [OMS] (2016). Planning and implementing palliative care services: a guide for pro- gramme managers. Institutional Repository for Information Sharing. https://apps. who.int/iris/handle/10665/250584

Organización Mundial de la Salud [OMS] (2018). Definition of palliative care. World Health Organization. http://www. who.int/cancer/palliative/definition/en/

Oudshoorn, S. (2015). En la cornisa de la vida. Intervenciones desde Terapia Ocupacional. Martin.

Phipps, K. y Cooper, J. (2014). A service evaluation of a specialist community palliative care occupational therapy service. Progress in Palliative Care, 22(6), 347351. https://doi.org/10.1179/1743291x1 4y.0000000104

Perilla, V. M. L. (2019). Caracterização da prática dos terapeutas ocupacionais em cuidados paliativos nos serviços públicos oncológicos de saúde no Brasil [Tesis de maestría, Universidade Federal de São Carlos]. Repositorio UFSCar. https:// repositorio.ufscar.br/handle/ufscar/11570

Pessini, L. y Bertachini, L. (2006). Nuevas perspectivas en cuidados paliativos. Acta Bioehtica, 12(2), 231-242. https://doi. org/10.4067/s1726-569x2006000200012

Pickens, N., Chow, J. K. y McKay, H. (2016). Role of Occupational Therapy in End-ofLife Care. The American Journal of Occupational Therapy, 70, 1-16. https://doi. org/10.5014/ajot.2016.706S17

Portela, S. G. y Galheigo, S. M. (2015). Cuidados Paliativos na atenção domiciliar: a perspectiva de terapeutas ocupacionais. Cadernos Brasileiros de Terapia Ocupacional, 23(1), 15-29. https://doi. org/10.4322/0104-4931.ctoao472 
Pyle, E. (2018). Analyzing the benefits of occupational therapy within palliative care for patients with cancer: A systematic review [Tesis de grado, Eastern Kentucky University]. Honors Theses. 538. https:// encompass.eku.edu/honors theses/538

Saunders, C. (1972). The care of the dying patient and his family. Contact, 39(1), $12-$ 18. https://doi.org/10.1080/13520806.19 72.11759235

Twycross, R. (2006). A tribute to dame Cicely Saunders. St Christopher's Hospice. https://www.stchristophers.org.uk/about/ damecicelysaunders/tributes

Tremblay, D., Roberge, D., Cazale, L., Touati, N., Maunsell, E., Latreille, J. y Lemaire, J. (2011). Evaluation of the impact of interdisciplinarity in cancer care. BMC health services research, 11(1), 144. https://doi. org/10.1186/1472-6963-11-144

Wainer, R. (2003). Vivir muriendo-morir viviendo. Construcción profesional de la "dignidad", la "esperanza" y el "aquí-ahora" en un equipo de cuidados paliativos [Disertación de Licenciatura, Universidad de Buenos Aires]. Repositorio FILO: UBA. http://antropologia.filo.uba. ar/sites/antropologia.filo.uba.ar/files/documentos/Wainer\%20-\%20Tesis.pdf

Witt Sherman, D., Maitra, K., Gordon, Y., Simon, S., Olenick, M., Barbara, S., Doherty,J., Hough, M., Randolph,M. y Singh, A. (2017). Illustrating and analyzing the processes of interprofessional collaboration: a lesson learned from palliative care in deconstructing the concept. Journal of PaIliative Medicine, 20(3), 227-234. https:// doi.org/10.1089/jpm.2016.0332 